\title{
Language Acculturation and Social Capital Among Migrants in James Town, Accra
}

\author{
John Kwame Boateng ${ }^{1} \quad$ Raymond Asare Tutu ${ }^{2}$ \\ 1.School of Continuing and Distance Education, College of Education, University of Ghana, PO Box LG 31, \\ Legon, Accra, Ghana \\ 2.Delaware State University, Dover, Delaware, USA
}

The research is financed by Delaware State University's Academic Enrichment Fund (Sponsoring information)

\section{Abstract}

This study examines the relative effects of language acculturation on social capital accumulation in the context of a poor urban neighborhood in Accra, Ghana. Acculturation is a fluid concept with multiple dimensions. Generally, it has been described as the extent to which migrants adopt the customs and values of their host populations. The fluidity of the concept has led to different ways of measurement. While a number of studies have assessed acculturation using dimensions such as assimilation, integration, marginalization, and separation to understand migrants lived experiences, there is paucity of research on the impact of language acculturation on social capital formation. Language barrier has been found to be associated with adaptation difficulties among migrants while higher language competency is associated with well-being factors such as lower anxiety and depression. In this study, in the internal migration context, we examine if Ga language competency predicts social capital accumulation among 301 migrants. Using a semi-structured survey questionnaire, we elicited information about migrants' fluency in the Ga language and social capital variables from which a social capital index was created. Using Chi-square tests and one-way ANOVA, at the bivariate level we fitted an ordinal logit regression model. We found that better Ga language fluency is associated with higher social capital. Religiosity (the extent to which the individual practices their religious beliefs) and marital status were significant predictors of social capital. It was concluded that low and high language acculturation was linked to poor and high self-rated health status of migrants in Jamestown.

Keywords: Blended mode, online, Sakai LMS, interactivity, student learning

DOI: $10.7176 / \mathrm{JLLL} / 68-06$

Publication date:May $31^{\text {st }} 2020$

\section{Introduction}

Acculturation is said to be a multidimensional process of change in behaviours, attitudes, and beliefs in both a newly arrived group and the dominant culture with which it interacts (Schwartz, Unger, Zamboanga \& Szapocznik, 2010). Kao (2006) noted that language acculturation has important implication in the formation of social ties and the acquisition of resources.

Migration remains a global phenomenon. In Ghana, over $50 \%$ of the population live outside the village from where they were born. Interregional movement is seen as a major determinant of where people live. Greater Accra region by far attracts more migrants (both internal and external migrants) than any other region in Ghana (Cassels, Jenness, Biney, Ampofo, \& Dodoo, 2014; Personal observation by authors). The general trend is that, people fleeing poverty from the less economically endowed locations in the northern parts of the country eventually end up in the relatively richer regions in the southern parts of the country. Greater Accra region is the chief among them. Many such migrants end up in poor neighbourhoods in the city's urban centres because they cannot afford rents in better and more expensive residential areas.

On the margins of society, the poor conditions they live in make them susceptible to environmentally induced diseases such as malaria, cholera and typhoid. The double burden of infectious and chronic diseases, wealth, income and other health factors also become important. Research has shown that, the socio-demographic factors, individual lifestyles and the type of resources migrants could access through their social networks played a part in how healthy or unhealthy migrants get (Tutu, Boateng, Ameyaw \& Busingye, 2016a \& b). Research had also shown that social relationships affect the social support networks migrants can rely on to access health care services. The association between poor migrants' lifestyles and their health in poor urban neighbourhoods as well as how social capital mediate that association has been largely a neglected area of research. Previous research in Ghana on poverty and health has largely examined the spatial distribution of inequalities in health. It has shown that, there is uneven distribution of diseases with some places having higher prevalence of certain diseases than others (Annim, Sebu \& Mariwah, 2012).

However, it is believed that, migrants who feel that they have sufficient language acculturation or use or will more likely have enough information on how to live successfully (including how to access health care and other network related health interventions) in their chosen environments - largely benefiting from the social networks that their language acculturation must have helped them to own. 


\subsection{Research Questions}

Two research questions asked are:

a. Do migrants who feel that they have sufficient language acculturation or use have enough information on how to live successfully including how to access health care and other networks related to health interventions in their chosen environments?

b. Do migrants with sufficient language acculturation or use largely benefit from the social networks that their language acculturation must have helped them to own?

\subsection{Literature Review}

The Acculturation Model of Berry (Berry et al., 2006) explains four preference classifications, namely, Assimilation, Separation, Integration and Marginalization. Cohen (2011) explains the four acculturation preference classifications as follows: Assimilation is where migrants coming from a different culture, adopts the cultural norms and heritage of the host community that they have migrated to. There is evidence that assimilation strategy has been observed to be the acculturation approach most preferred by host communities (Horverak et al., 2013; Kosie et al., 2005). Separation is said to have occurred when migrants rejects the culture of the host community and sticks to their own culture of origin. Integration occurs, when migrants adopts both the host community's cultural norms and heritage and their own cultural norms and heritage combining the two at the same time.

According to Arends-Toth \& van de Vijver, (2003), migrants prefer integration in the public sphere and a separation strategy is preferred in their private circles. When someone rejects their own original culture and heritage and that of the host community, marginalization is said to have occurred. Horverak et al., (2013) observed that marginalization preference is related to individualistic traits such as anxiety, closed-mindedness and unsociability. Mondain and Lardoux (2013) observed that a wide range of socio-contextual factors and personal characteristics have been identified to influence acculturation. Research by Mondain and Lardoux has further shown that, international migrants' extent of integration is a function of the reason for migration, socio-economic status, educational background and proximity to spaces and places for practicing social and cultural norms such as language and marriage ceremonies of their countries of origin.

At the individual level, Kalmijn \& van Tubergen, (2006) showed that personal traits such as age, migration, educational attainment, duration of stay at the destination are essential for acculturation. Braun \& Glockner-Rist, (2012) added that, previous migration experience is also important for acculturation. Furthermore, Djamba \& Kimuna, (2012) showed that, at the societal level, place of settlement affects acculturation in ways related to job market opportunities and constraints.

Social capital is important for the acculturation process to happen. Awumbila et al., (2016), illustrated social capital in different forms and types such as bonding, bridging and linking. According to Hawkins \& Maurer (2010), bonding social capital refers to strong horizontal ties between individuals or groups sharing similar sociodemographic features. Family and immediate relations ties demonstrate such bonding relationships and are good for significantly enhancing integration.

According to Tutu et al., (2016), bridging social capital denotes ties outside immediate family but cuts across different individuals. This form of social capital is considered to project weak ties, includes membership in associations and contact with friends. Benefits that associates with bridging social capital are by virtue of rights and privileges attached to being part of a group or union. Linking social capital refers to vertical social ties, signifying different social power relations (Tutu et al., 2016). Pieterse (2003), refers to this as "friends in high places". Social networks facilitates bonding and bridging social capital. Tutu et al., (2016), observed that, social networks manifested in associational membership through clubs, fellowship, and unions are major reservoirs for social capital due to mutually shared goals, interests and benefits.

Raza et al., (2013) provide a modification of Berry's Acculturation Model. In this modification, Raza et al., (2013) revealed that, individualization which signifies the experience of weak bonding and weak bridging network ties by migrants is as a result of experiencing cultural isolation or the denunciation of cultural attributions associated with marginalization classification of acculturation. Strong bonding and strong bridging social capital are exhibited in integration while separation shows strong bonding and weak bridging social capital formation. Assimilation depicts weak bonding and strong bridging. According to Portes et al., (2009), even in the context of closed ethnic communities, social capital facilitates assimilation and Jun \& Hu (2015) reported that strong bridging social capital, which has been found to be a function of longer duration of stay at the destination, enhances migrants' social integration. The interest in this paper is language acculturation and social capital. We examined the relative effects of language acculturation on social capital accumulation and questions whether the Ga language competency predicts social capital accumulation?

\subsection{Methodology}

3.1 Study Area

Jamestown is located east of the Korle Lagoon. Jamestown is one of the oldest districts in the city of Accra. The 
town emerged as communities around the $17^{\text {th }}$ century British James Fort and Dutch Ussher Fort on the Gulf of Guinea Coast. The community became an area of dense mixture of commercial and residential use. Now, Jamestown remains a fishing community inhabited by Ga people and many others from all parts of Ghana. The town remains in a state of decay following years of neglect by subsequent governments, and the district is a popular tourist destination for those seeking to see the remnants of Accra's colonial past.

\subsection{Data and Methods}

\subsubsection{Dependent variable}

The Ghana Statistical Service divides communities into Enumeration Areas (EAs) for the purpose of National Census. Jamestown, the current study area happens to have twenty of such EAs with a total population of 15, 508 . Cross-tabulating the population in each area by Ethnicity, Tutu et al., (2016) found the following ethnic categories: Akan, Ga-Adangme, Ewe, Guan, Gurma, Mole-Dagbani, Grusi, Mande and others. According to Tutu et al., (2016), The Ga-Adangme population totaled 8,935 and the non-Ga population totaled 6,573. The sample size for the current study was 363 using the statistical software Epi info 7 for the determination and producing the following power calculation indicators: Limit (5\%); design effect (1); cluster (1); effect size (50\%); and confidence level of $95 \%$. A proportional number of households in conformity with the size of the population in each EA was selected. In total, 301 migrants participated in the current study. Four trained research assistants administered the semistructured questionnaire tool over a five-week period.

The dependent variable, Social Capital focused on actual resources received by participants. Social capital index (low, medium and high) was employed. And "yes" and "no" responses were solicited to the following questions: "do you receive, material (money, cloth, food, toiletries and so on) help from the Gas?" "Do you receive information (facts, knowledge) about how to live successfully here from the Gas?" Do you receive emotional help from the Gas here (e.g. cheer you up when you are sad, give you confidence in life, spend time with you, and so on?"

\subsubsection{Independent variables}

The main predictor variable was "Ga language competency". Respondents were asked to rate their competency in Ga on the basis of "not at all"; "somewhat"; "fluent"; "very fluent". The socio-demographic predictors employed included sex, educational level, marital status, employment status, religious affiliation, religiosity and age.

\subsubsection{Data Analyses}

A two-stage analytical procedure was followed. The first stage entailed bivariate analysis comprising a Chi-Square test and a one-way ANOVA. The second stage was a multivariate analysis employing an Ordinal Logistic Regression.

\subsection{Results and Discussion}

Results from the bivariate analysis in the table below (Table 1) investigates the relationship between various demographic status of respondents and social capital. One-way ANOVA has been used to investigate how each of these demographic characteristics affects social capital. At 0.05 level of significance, it is indicated that respondent's level of fluency in Ga, their marital status and their religiosity significantly influenced social capital formation. However, the test statistics for sex, educational level, employment status, religious affiliation, income per week and age showed no statistical significance with social capital. It is inferred that these demographic variables do not affect social capital, however the previous does.

Table 1. Bivariate Analysis (X2 and one-way ANOVA)

\begin{tabular}{l|l}
\hline Variable & Values \\
\hline How would you rate your fluency in $\mathrm{Ga}$ & $14.19^{*}$ \\
\hline sex & 1.52 \\
\hline Educational Level & 11.32 \\
\hline Marital status & $24.22^{* * *}$ \\
\hline Employment Status & 5.28 \\
\hline Religious affiliation & 10.61 \\
\hline Religiosity & $59.44^{* *}$ \\
\hline Income per week (in Ghana Cedis) & 0.535 \\
\hline Age & 0.719 \\
\hline
\end{tabular}

$* * * \mathrm{p}<0.000 ; * * \mathrm{p}<0.005 ; * \mathrm{p}<0.05 ; \mathrm{R} 2-0.026$

Haven't identified that these categorical variables (Ga language competency, religiosity and marital status) are significantly related to social capital, a post-hoc test is run to identify the category of these variables that significantly predict social capital. The ordinal logistic regression has been used to investigate this assertion.

The standard interpretation of the ordered coefficient is that for a one unit increase in the predictor, the response variable level is expected to change by its respective regression coefficient in the ordered log-odds scale 
while the other variables in the model are held constant.

It is indicated that a one unit increase in Ga language competency, social capital is expected to change by $63.3 \%$ when a person is "not at all" competent in Ga in the ordered log-odds scale than when language competency is "very often" while all other variables were held constant. There is a strong association between Ga language competency and social capital. There is a significant and negative coefficient for Ga language competency "Not at all" and "somewhat". Taking the exponent of logits will give that of the default or reference group in an increased in social capital. It is can be inferred that there will be an increase in social capital when language competency is "not at all" than when language competency is "very often" exp ( -0.633$), 0.533$, greater than half.

It is indicated that a one unit increase in Ga language competency, social capital is expected to change by 95.4\% when a person is "somewhat" competent in Ga in the ordered log-odds scale than when language competency is "very often" 0.385 , less than half, while all other variables were held constant.

Table 2. Ordinal Logistic Regression (Ga Language Competency and Social Capital)

\begin{tabular}{llll}
\hline Variable & & $95 \%$ Confidence interval & \\
Ga Language competency & Estimates & Lower & Upper \\
Not at all & $-0.633^{*}$ & -1.575 & 0.308 \\
Somewhat & $-0.954^{* *}$ & -1.613 & -0.295 \\
Fluent & -0.306 & -1.029 & 0.416 \\
\hline
\end{tabular}

Very often (Ref)

$* * * \mathrm{p}<0.000 ; * * \mathrm{p}<0.005 ; * \mathrm{p}<0.05 ; \mathrm{R} 2=0.026$

The focus of this analysis (Table 2) is to explore the religiosity group differences in social capital status, understanding which religiosity affects higher social capital status. It is can be inferred that there will be an increase in social capital when religiosity is "occasionally" than when language competency is "always". It is indicated that a one unit increase in religiosity, social capital is expected to change by 1.502 when a person is religiosity is "occasionally" than when it is "always" while all other variables were held constant. This is also true for the following categories of religiosity: less often, often and very often.

Table 3. Ordinal Logistic Regression (Religiosity and Social Capital)

\begin{tabular}{|l|l|l|l|}
\hline Variable & \multirow{2}{*}{} & \multicolumn{2}{|l|}{$95 \%$ Confidence interval } \\
\cline { 3 - 4 } Religiosity & Estimates & Lower & Upper \\
\hline Not at all & -0.711 & -0.431 & 1.853 \\
\hline Occasionally & $1.502^{*}$ & 0.362 & 2.641 \\
\hline Less Often & $1.859^{* * *}$ & 1.054 & 2.736 \\
\hline Often & $1.933^{* * *}$ & 1.156 & 2.710 \\
\hline Very Often & $1.616^{* * *}$ & 0.865 & 2.367 \\
\hline Always (Ref) & & & \\
\hline
\end{tabular}

$* * * \mathrm{p}<0.000 ; * * \mathrm{p}<0.005 ;{ }^{*} \mathrm{p}<0.05 ; \mathrm{R} 2=0.26$

The focus of this analysis (Table 3 ) is to explore the marital status group differences in social capital status, understanding which marital status affects higher social capital status. It is can be inferred that there will be an increase in social capital when marital status is "divorces" than when marital is "married". It is indicated that in a unit increase in marital status, social capital is expected to change by 1.190 when a person's marital status is "divorces" while all other variables were held constant.

Table 4. Ordinal Logistic Regression (Marital status and Social Capital)

\begin{tabular}{|l|l|l|l|}
\hline \multicolumn{3}{|l|}{} \\
\cline { 1 - 1 } Variable & & $95 \%$ Confidence interval \\
\cline { 3 - 4 } Marital status & Estimates & Lower & Upper \\
\hline Single & 0.503 & -0.219 & 1.224 \\
\hline Consensual Union & -0.729 & -1.866 & 0.407 \\
\hline Divorces & $-1.190^{*}$ & -2.117 & -0.263 \\
\hline Separated & 0.012 & -1.004 & 1.029 \\
\hline Married (Ref) & & & \\
\hline
\end{tabular}

$* * * \mathrm{p}<0.000 ; * * \mathrm{p}<0.005 ; * \mathrm{p}<0.05 ; \mathrm{R} 2=0.26$

The result has implied that being fluent in the Ga language, being married as in one's marital status and religiosity (going to religious services always) leads to social capital accumulation which are essential for social connectivity. This finding, seems to lend support to Kim et al' (2014) observation about strong family ties by migrants through marriage and social capital. Other socio-demographic factors influencing social capital accumulation showed some unconventional findings. For instance, sex, educational level, employment status, religious affiliation, income per week and age showed no statistical significance with social capital accumulation. 
One would have taught that, one's religious affiliation, or his/her employment status or his/her educational level or even sex would have influence somehow his or her social connectivity.

\subsection{Conclusion}

This study has examined the relative effects of language acculturation on social capital accumulation in the context of a poor urban neighborhood in Accra, Ghana. Acculturation is a fluid concept with multiple dimensions. Language barrier has been found to be associated with adaptation difficulties among migrants while higher language competency is associated with well-being factors such as lower anxiety and depression. In this study, in the internal migration context, we examine if Ga language competency predicted social capital accumulation among 301 migrants. We conclude that, language barriers was associated with adaptation difficulties and that, language competency is associated with social capital accumulation implying well-being factors such as lower anxiety and depression. Likewise, low and high language acculturation was associated with poor and high selfrated health of migrants in Jamestown.

\subsection{References}

Annim, S. K. Sebu, J \& Mariwah, S. (2012). Spatial Inequality and Household Poverty in Ghana, Economic Systems 36(4):487 - 505 - DOI: 10.2139/ssrn.1983340

Awumbila M., Teye JK., and Yaro JA (2016). Social Networks, migration trajectories and livelihood strategies of migrant domestic and construction workers in Accra, Ghana. Journal of Asian and African Studies, Epub ahead of print 17 March. DO1 10.1177/0021909616634743.

Arends-Toth, JV and van de Vijver, FJR (2003). Multiculturalism and acculturation: Views of Dutch and TurkishDutch. European Journal of Social Psychology. Vol 33, pp. 249-266. Doi. 10.1002/ejsp.143

Berry J.W., Sam, D. L., Phinney, J.S., Vedder, P. (2006). Immigrant Youth: Acculturation, Identity and Adaptation, Applied Psychology Vol 55, Issue 3 pp 303 - 332

Braun M., and Glockner-Rist, A. (2012). Patterns of social integration of Western European Migrants. Journal of International Migration and Integration. Vol 13, Issue 4, pp 403-422.

Coleman, J. S. (1988). Social Capital in the Creation of Human Capital. The American Journal of Sociology 94, pp95-120

Cassels, S., Jenness, S. M., Biney, A. A., Ampofo, W. K., \& Dodoo, F. N. (2014). Migration, sexual networks, and HIV in Agbogbloshie, Ghana. Demographic research, 31, 861-888. https://doi.org/10.4054/DemRes.2014.31.28

Djamba YK, and Kimuna SR. (2012). The labor force participation and earnings gap among African immigrant women in the USA. Journal of International Migration and Integration, Vol 13, Issue 4 pp $481-501$.

Hawkins RR, and Maurer K (2010). Bonding, Bridging and linking: How social capital operated in New Orleans following Hurricane Katrina. British Journal of Social work, Vol 40, Issue 6, pp1777 - 1793.

Horverak JM, Sandal GM, Pallesen S and Timmerman, ME (2013). Hiring rankings of immigrant job applications: Immigrants acculturation strategies and managers personality trait perception. Journal of Journal of International Migration and Integration. Vol 14, Issue 3, pp 493-510.

Jun HJ and Ha SK (2015). Social Capital and assimilation of migrant workers and foreign wives in South Korea.: The case of Wongok community, Habitat International, Vol 47, Issue 1 pp 126-135.

Kalmijn M, and van Tubergen F (2006). Ethnic intermarriage in the Netherlands: Confirmations and refutations of accepted insights. European Journal of Population, Vol 22, Issue 4, pp 371-397.

Kao G (2006). Parental Influences on the educational outcomes of immigrant youth. International Migration Review, Vol 38, Issue 2 pp 427 - 449.

Kim, J. Heo, J. and Park, S.H (2014). The exploration of acculturation and health among immigrants from noneastern cultures, Qualitative Health Research, Vol 24, Issue 8 pp 1138-1149.

KosicA, Mannetti L. and Sam DL (2005). The role of majority attitudes towards out-group in the perception of the acculturation strategies of immigrants. International Journal of Intercultural Relations. Vol 29, Issue 3 pp 273-288.

Mondain N, and Lardoux S (2013). Transitions to adulthood among first generation sub-Saharan African immigrant addolescents in Canada: Evidence from a qualitative study in Montreal. Journal of International Migration and Integration, Vol 14, Issue 2, pp 307-326.

Pieterse JN (2003). Social capital and migration beyond ethnic economics. Ethnicities Vol 3, Issue 1 pp 29-58.

Portes A., Fernandez-Kelly P, and Haller W (2009). The adaptation of the immigrant second generation in America: A theoretical overview and recent evidence. Journal of Ethnic and Migration Studies, Vol 35, Issue 7 pp 1077-1104.

Raza M., Beaujot R and Woldemicael G. (2013). Social capital and economic integration of visible minority immigrants in Canada. Journal of International Migration and Integration, Vol 14, Issue 2, pp263-285.

Schwartz, S.J., Unger, J.B., Zamboanga, B.L., Szapocznik J. (2010). Rethinking the concept of acculturation: 
implications for theory and research. Am Psychol. 65 (4): pp 237 -251, doi: 10.1037/a0019330

Tutu, R.A., Boateng, J., Ameyaw E.E., Busingye J.D., (2016). Acculturation inclinations and subjective health status of internal migrants in James Town, an urban slum settlement in Accra. Springer - Journal of Population Researcch, Doi: 10.1007/s12546-016-9182-z

Tutu, R.A., Boateng, J., Ameyaw E.E., Busingye J.D., (2016). Togetherness in difference": Perceived Personal Discrimination and Acculturation Preferences. Among Internal Migrants in Poor Urban Community in Accra. Journal of Asia and African Studies (JAAS), November, pp 1-17 